\title{
MANUTENÇÃO DE LOCOMOTIVAS - TRACK SERVICE*
}

\author{
Gustavo Elói de Sá Lima ${ }^{1}$ \\ Juliano Viana Batista ${ }^{2}$ \\ Lúcio Flávio Drummond Matos ${ }^{3}$ \\ Jesus Jonatan Souza Santos ${ }^{4}$
}

\section{Resumo}

A estratégia de manutenção de locomotivas da VLI sofreu significativa modificação após a implementação de propostas de consultores americanos, baseadas principalmente, na execução de atividades fora de oficina, realizadas em estruturas chamadas de "Track Service", estruturas as quais contam basicamente com uma vala para inspeção na parte inferior das locomotivas. Dessa forma, foi possível capturar diversos ganhos em custeio e redução de investimentos, como por exemplo o aumento de periodicidade entre manutenções preventivas e redução do projeto da nova oficina de Paulínia.

Palavras-chave: Manutenção; Estratégia; Locomotivas; Track Service.

\section{Abstract}

\section{LOCOMOTIVE MAINTENANCE - TRACK SERVICE}

VLI's locomotive maintenance strategy has undergone significant change after the implementation of American consultants proposals, mainly based in the execution of activities outside workshop, held in structures called "Track Service", structures that have a ditch for inspection at the bottom of the locomotives. In this way, it was possible to capture several gains in costing and reduction of investments, such as the increase of periodicity between preventive maintenance and reduction of the project of the new Paulínia workshop.

Keywords: Maintenance, Strategy, Locomotives; Track Service.

1 Engenheiro Mecânico, pós-graduado em Engenharia Ferroviária, Analista de Engenharia, Engenharia de Material Rodante, Divinópolis, Minas Gerais, Brasil.

2 Engenheiro de Produção, pós-graduado em Engenharia de Manutenção, Especialista de Engenharia, Engenharia de Material Rodante, Divinópolis, Minas Gerais, Brasil.

3 Engenheiro de Controle e Automação, pós-graduado em Engenharia Ferroviária, Analista de Engenharia, Engenharia de Material Rodante, Belo Horizonte, Minas Gerais, Brasil.

4 Engenheiro Eletricista, Mestre em Engenharia Elétrica, Doutorando em Engenharia Elétrica, Supervisor de Engenharia de Vagões, Divinópolis, Minas Gerais, Brasil 


\section{INTRODUÇÃO}

A equipe de engenharia, juntamente com os consultores americanos, realizou visitas técnicas nas estruturas da ferrovia norte americana BNSF durante duas semanas de outubro de 2014. Durante a visita foi bastante debatida a aplicação de atividades de manutenção de locomotiva fora de oficina, utilizando uma estrutura dotada de vala, externa à oficina, preferencialmente próxima à linha principal de circulação ou até mesmo no próprio posto de abastecimento, assim o tempo de imobilização da locomotiva ou trem é reduzido, além de minimizar a quantidade de manobras necessárias para posicionamento da máquina.

O objetivo do "Track Service" é o aproveitamento do tempo ocioso durante o abastecimento ou formação de trens para a realização de inspeções e atividades preventivas rápidas, além da execução de pequenas corretivas. Resumindo o escopo de trabalho no Track Service, conforme os próprios consultores americanos: "Todas as atividades que durem menos de 2 horas e sejam possíveis de serem realizadas apenas com a caixa de ferramentas". É importante ressaltar que para um maior ganho de produtividade das equipes são necessárias estruturas auxiliares à vala, como container com materiais, ferramentas e computador com acesso à rede da ferrovia e aos sistemas de monitoramento remoto das locomotivas (Intellitrain - EMD e LMS GE).
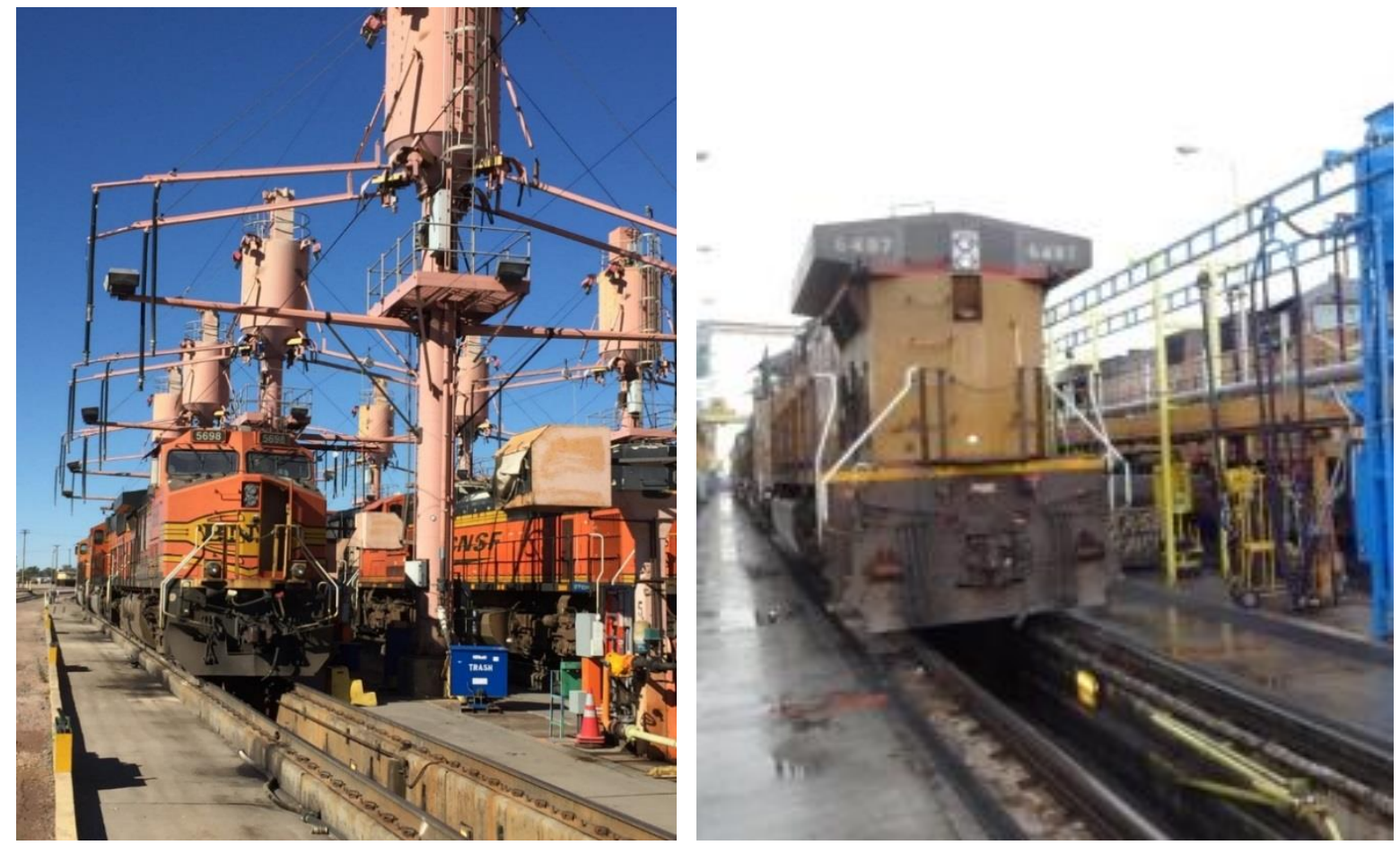

Figura 01. Track Service e Container de Materiais BNSF. 

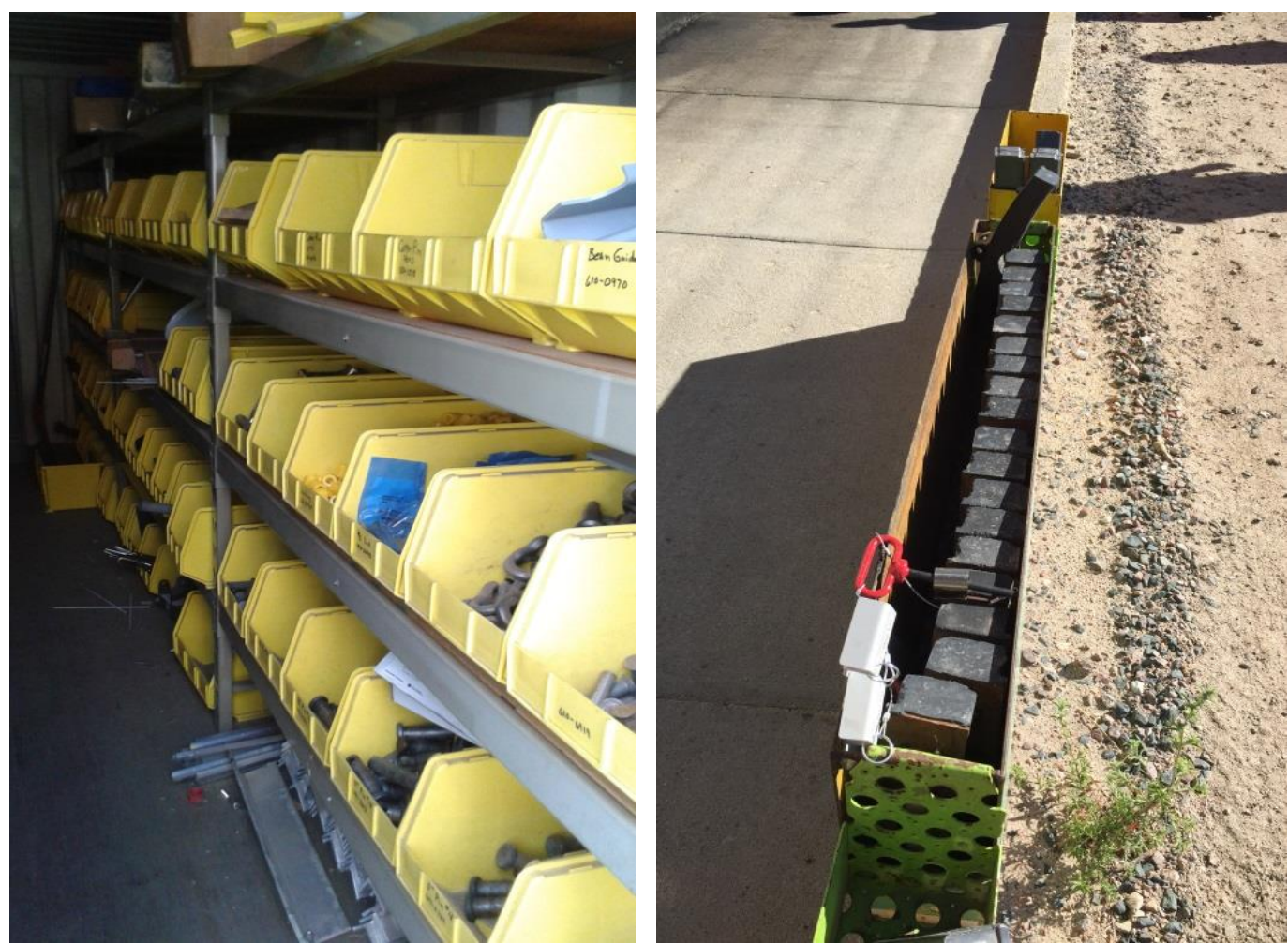

Figura 02. Materiais dentro do container e dispostos ao longo da vala da BNSF.

Ao retornar, a equipe de engenharia direcionou os estudos para desenvolvimento e implantação do modelo de manutenção "Track Service".

O estudo inicial foi realizado no corredor Centro Sudeste, mais especificamente nas instalações de Paulínia, devido a oportunidade de ajuste no projeto da nova oficina, do novo pátio e novo posto de abastecimento.

\section{MATERIAIS E MÉTODOS}

Todo o trabalho foi desenvolvido com base em simulações computacionais, cujos modelos estatísticos foram construídos através da teoria das filas, sendo capazes de descrever as taxas de ocupação dos pátios para diversos cenários.

\section{RESULTADOS E DISCUSSÃO}

O primeiro passo foi a simulação operacional para definição da localidade a ser construída a estrutura de vala "Track Service", o objetivo do estudo foi determinar o local que geraria o menor impacto operacional, do ponto de vista de tempo de ciclo, formação de fila na entrada do pátio e formação de fila na entrada do posto de abastecimento, além de definir a melhor periodicidade entre as manutenções, sabendo que o tempo para execução das atividades não poderia ser superior a 2 horas.

O resultado da simulação operacional indicou que a o intervalo ideal entre as manutenções em "Track Service" deveria ser de 30 dias e a melhor localização da estrutura de vala seria fora do posto de abastecimento, na mesma linha, porém disposto posteriormente aos bicos de abastecimento. 
Tabela 01. Resultado simulação operacional

\begin{tabular}{|c|c|c|c|c|c|c|c|c|}
\hline & \multirow[b]{2}{*}{ Track Service (TS) } & \multirow[b]{2}{*}{ Intervalo entre TS } & \multirow[b]{2}{*}{ Local do TS } & \multirow[b]{2}{*}{$\begin{array}{c}\text { Tempo de } \\
\text { abastecimento }\end{array}$} & \multirow[b]{2}{*}{$\begin{array}{c}\text { Abastecimento no } \\
\text { tempo morto do } \\
\text { TS? } \\
\end{array}$} & \multicolumn{3}{|c|}{ Resultado } \\
\hline & & & & & & $\begin{array}{l}\text { THP ZOP } \\
\text { (h) }\end{array}$ & $\left|\begin{array}{c}\text { Fila } \\
\text { posto (h) }\end{array}\right|$ & $\begin{array}{l}\text { Fila TS } \\
\text { (h) }\end{array}$ \\
\hline Cenário 0 & Não & - & - & 30 a $60 \mathrm{~min}$ & - & 7,5 & 0,3 & - \\
\hline Cenário 1 & Sim & 15 dias & PA & 30 a $60 \mathrm{~min}$ & Sim & 7,9 & 1,3 & 0,9 \\
\hline Cenário 2 & Sim & 15 dias & aolado do PA & 30 a $60 \mathrm{~min}$ & Não & 7,7 & 0,4 & 0,3 \\
\hline Cenário 3 & Sim & 15 dias & $\mathrm{PA}$ & 30 a $60 \mathrm{~min}$ & Não & 9,5 & 3,5 & 2,1 \\
\hline Cenário 4 & Sim & 15 dias & PA & 13 a $18 \mathrm{~min}$ & Sim & 7,6 & 0,7 & 0,5 \\
\hline Cenário 5 & Sim & 15 dias & ao lado do PA & 13 a $18 \mathrm{~min}$ & Não & 7,3 & 0,1 & 0,3 \\
\hline Cenário 6 & Sim & 30 dias & PA & 30 a $60 \mathrm{~min}$ & Sim & 7,7 & 0,5 & 0,5 \\
\hline Cenário 7 & Sim & 30 dias & aolado do PA & 30 a $60 \mathrm{~min}$ & Não & 7,7 & 0,3 & 0,1 \\
\hline
\end{tabular}

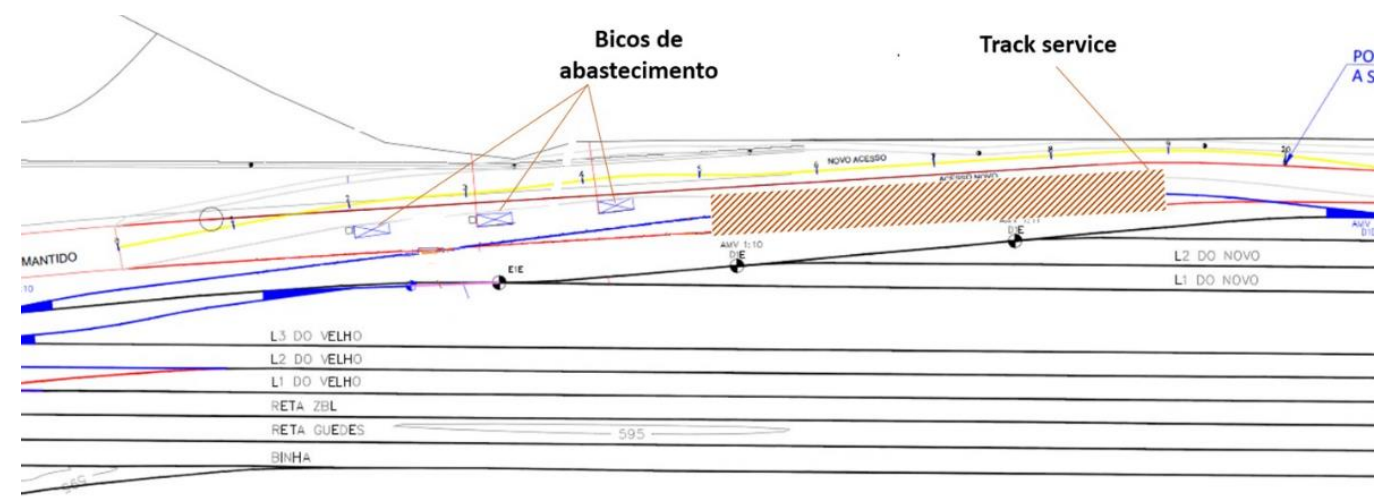

Figura 03. Materiais dentro do container e dispostos ao longo da vala da BNSF.

Com o resultado da simulação em mãos, o próximo passo foi a revisão da estratégia de manutenção de locomotivas. Utilizando o intervalo de 30 dias para a realização das atividades "Track Service" juntamente com uma inspeção de escopo menor (ITS Inspeção Track Service), foi possível aumentar a periodicidade das Inspeções de Conservação (IC) das locomotivas de maior potência que operam nos fluxos que passam por Paulínia. Na tabela 02 é possível entender a alteração na estratégia de manutenção.

Tabela 02. Comparativo entre estratégias

\begin{tabular}{|c|c|c|c|}
\hline \multicolumn{2}{|c|}{ PLANO } & $\begin{array}{c}\text { ESTRATÉGIA } \\
\text { ANTIGA }\end{array}$ & $\begin{array}{c}\text { ESTRATÉGIA } \\
\text { NOVA }\end{array}$ \\
\hline \begin{tabular}{c|c|c|c|} 
Periodicidade \\
(dias)
\end{tabular} & ITS & - & 30 \\
\hline \multirow{2}{*}{ IC } & 90 & 180 \\
\hline HH necessário & ITS & - & 2 \\
\hline & IC & 24 & 24 \\
\hline
\end{tabular}

A partir desta alteração na estratégia foi possível capturar ganhos no processo de manutenção (OPEX) com a redução de $\mathrm{HH}$ e redução de consumo de materiais, além da redução no ciclo dos trens, gerando um ganho em desinvestimento de uma locomotiva no fluxo. 
Um outro ganho bastante expressivo em CAPEX foi a oportunidade de revisão do projeto da nova oficina de Paulínia, uma vez que a necessidade de slots para a manutenção seria reduzida, devido ao aumento da periodicidade dos planos de manutenção IC das locomotivas de maior potência. No projeto inicial, a oficina teria 18 slots para manutenção de locomotivas, após a revisão esse número caiu para 12 slots, sendo possível retirar toda uma linha de dentro da oficina. A modificação pode ser vista na figura 04.

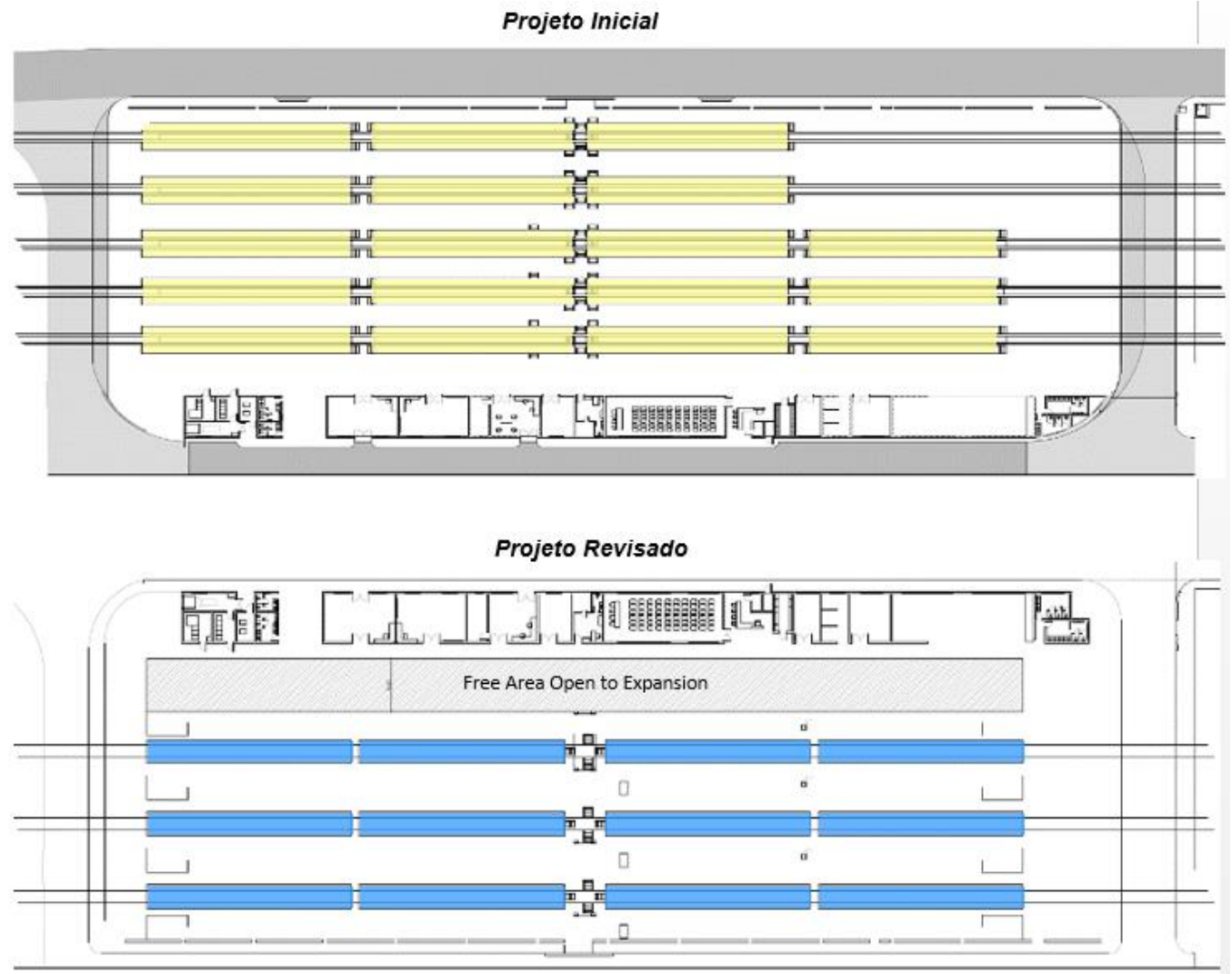

Figura 04. Revisão do projeto da nova oficina de Paulínia.

Com a definição da localidade feita através da simulação, foi contratada a empresa responsável pela construção da estrutura do "Track Service" conforme o projeto conceitual elaborado pela VLI.

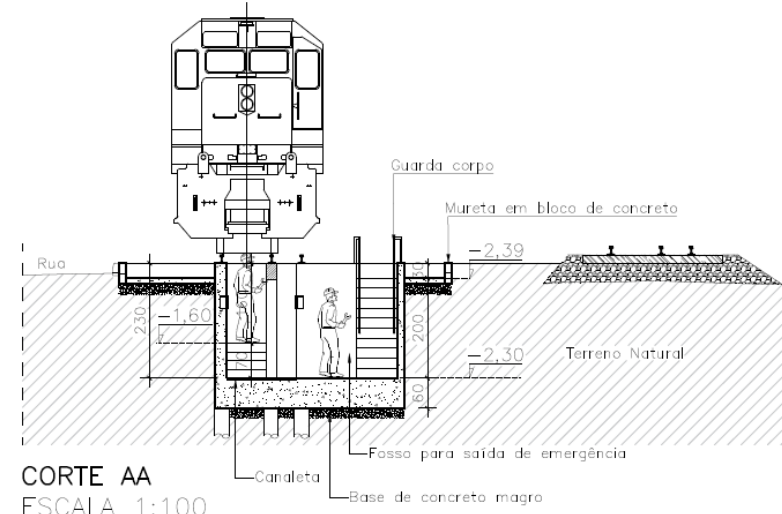

Figura 05. Conceitual da estrutura "Track Service" de Paulínia. 
A empresa contratada iniciou as obras da estrutura em abril de 2016 e concluiu a construção em julho de 2016.

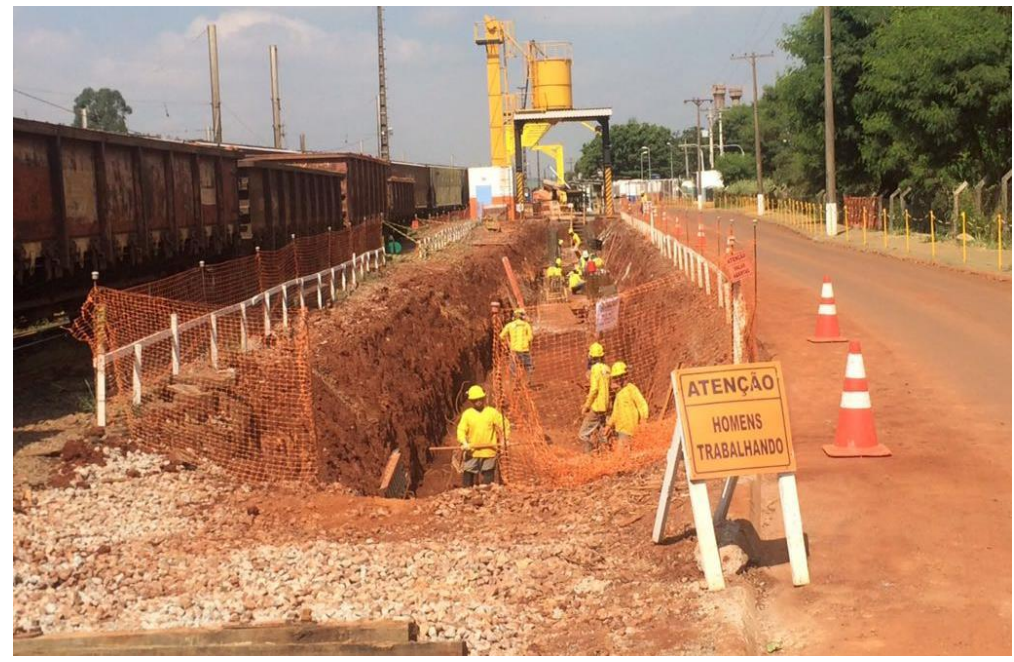

Figura 06. Obra em fase inicial.
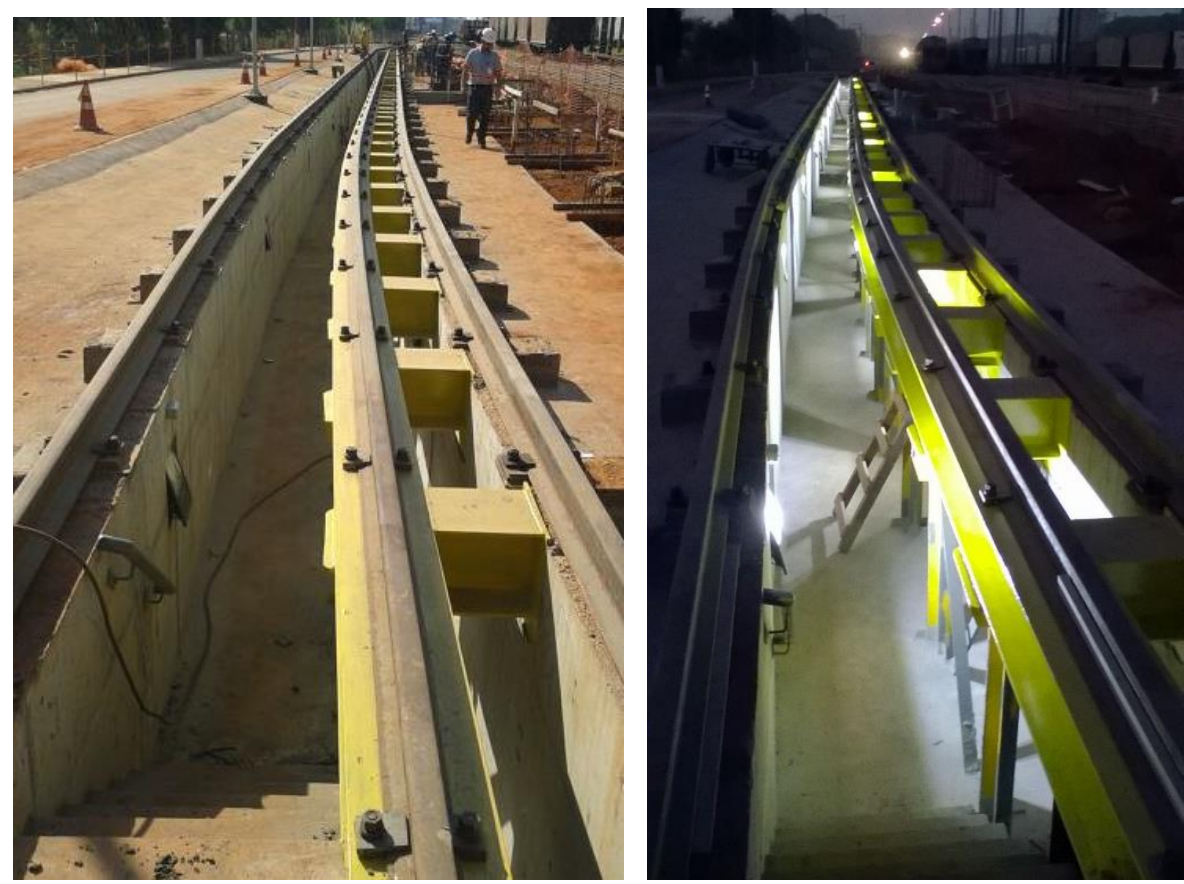

Figura 07. Obra finalizada.

Após a finalização das obras, a área de manutenção de locomotivas juntamente com a engenharia, chegaram a um consenso de que seriam necessárias adequações na estrutura do "Track Service" para que a execução dos serviços de manutenção pudesse ocorrer sem risco de segurança aos colaboradores. Assim, em agosto de 2016 a instalação de postes de iluminação e de uma linha vida foi finalizada. 


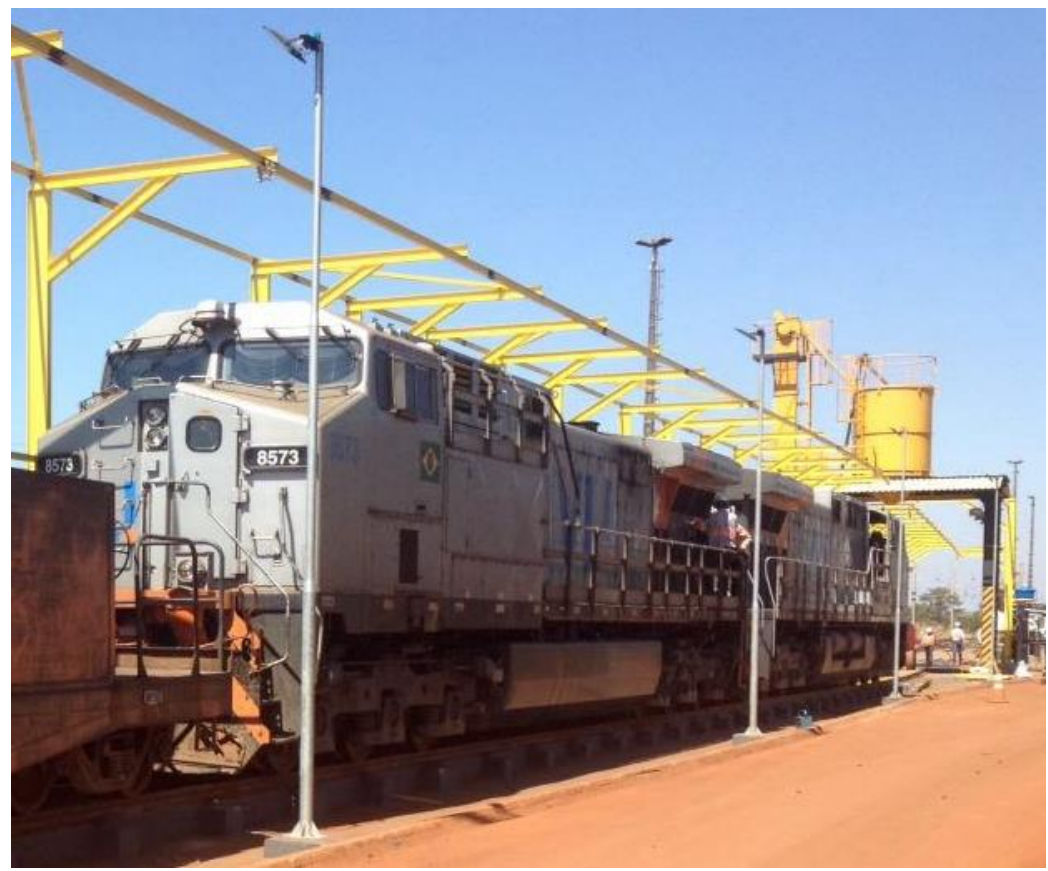

Figura 08. Adequações finalizadas.

Como estrutura de apoio às atividades do "Track Service", foi também disponibilizado um container ao lado da linha. O container conta com um ponto de rede e um toughbook que permite aos colaboradores acessarem a rede de computadores VLI além de um espaço dedicado ao armazenamento de ferramentas e de materiais de alto consumo.

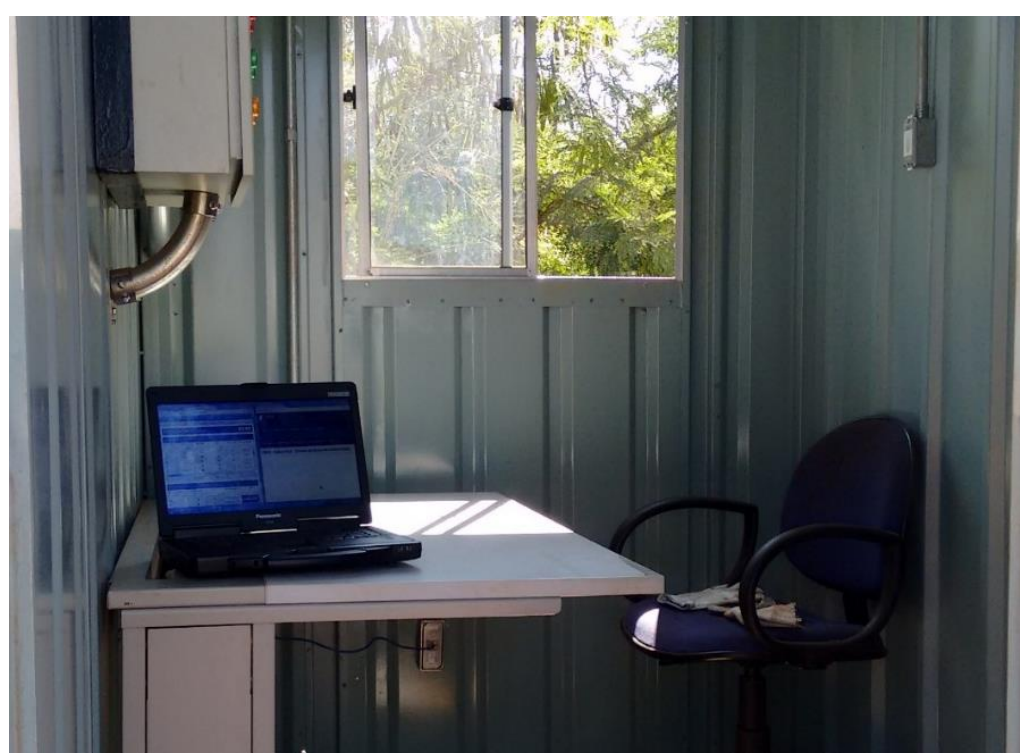

Figura 09. Toughbook no container com acesso a rede. 


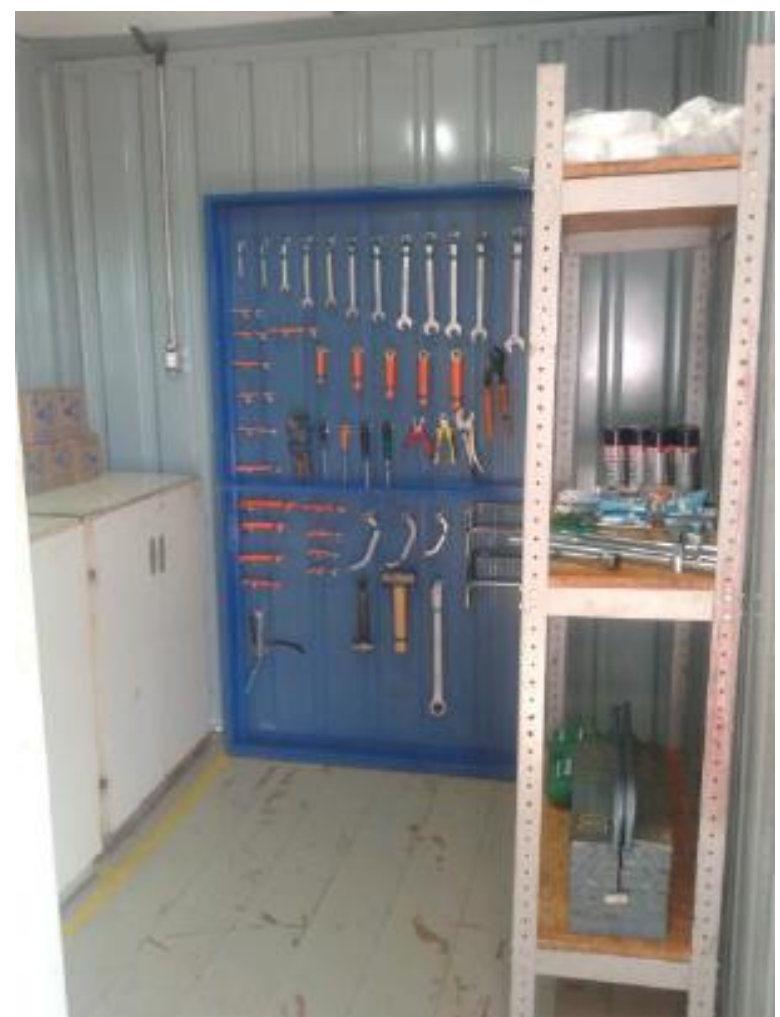

Figura 10. Ferramentas e materiais armazenados no container.

Anteriormente e paralelamente à construção da vala em Paulínia, diversas ações de estruturação dos processos que sustentarão as atividades no "Track Service" foram realizadas.

Com a definição do tempo de duas horas para execução das atividades e sabendo da periodicidade de 30 dias a cada intervenção no "Track Service", foi necessário elaborar o check list da inspeção preventiva a ser realizada na vala fora de oficina, de forma a sustentar o aumento para 180 dias do intervalo de realização da inspeção IC.

O check list da inspeção ITS foi elaborado para as frotas AC44, SD70, SD70BB e GT46. A premissa inicial foi de que o tempo necessário para a realização da ITS seria de uma hora, assim das duas horas de imobilização da locomotiva no "Track service", uma hora seria para a execução do check list preventivo ITS e a outra hora estaria disponível para a execução de ordens de serviço corretivas, lembrando que devido a limitação de estrutura, nem todas corretivas são passíveis de execução na estrutura da vala. O check list da ITS foi elaborado inicialmente com 58 atividades.

Com o check list definido e cadastrado no sistema, o próximo passo foi o treinamento dos executantes selecionados para trabalhar no "Track Service", conforme evidência da figura 11. O treinamento foi realizado em duas etapas, uma teórica e outra prática na própria estrutura recém construída. 


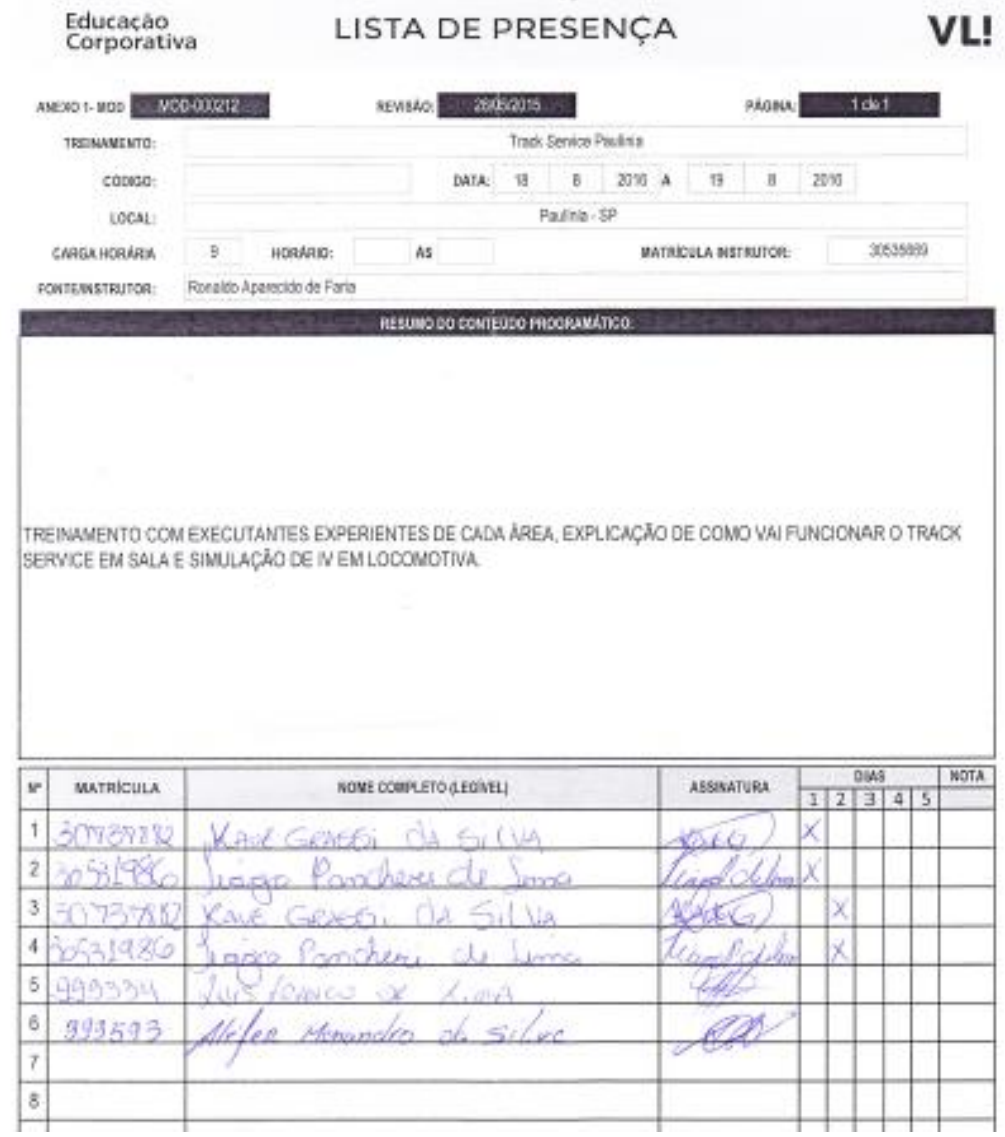

Figura 11. Treinamento execução da ITS

Por se tratar de um novo processo, em que a agilidade de execução é muito importante, o processo de PCM deve funcionar em perfeita sintonia com a execução, de forma que não faltem recursos, como materiais e componentes, durante as duas horas em que a locomotiva estiver disponível no "Track Service". Dessa maneira, foi elaborado um padrão de PCM específico para a ITS e as atividades relacionadas ao "Track Service".

O padrão conta com um fluxograma das principais etapas de planejamento e programação dos serviços realizados no "Track Service", além da definição das responsabilidades, entradas e saídas de cada função do PCM em todo o processo. 

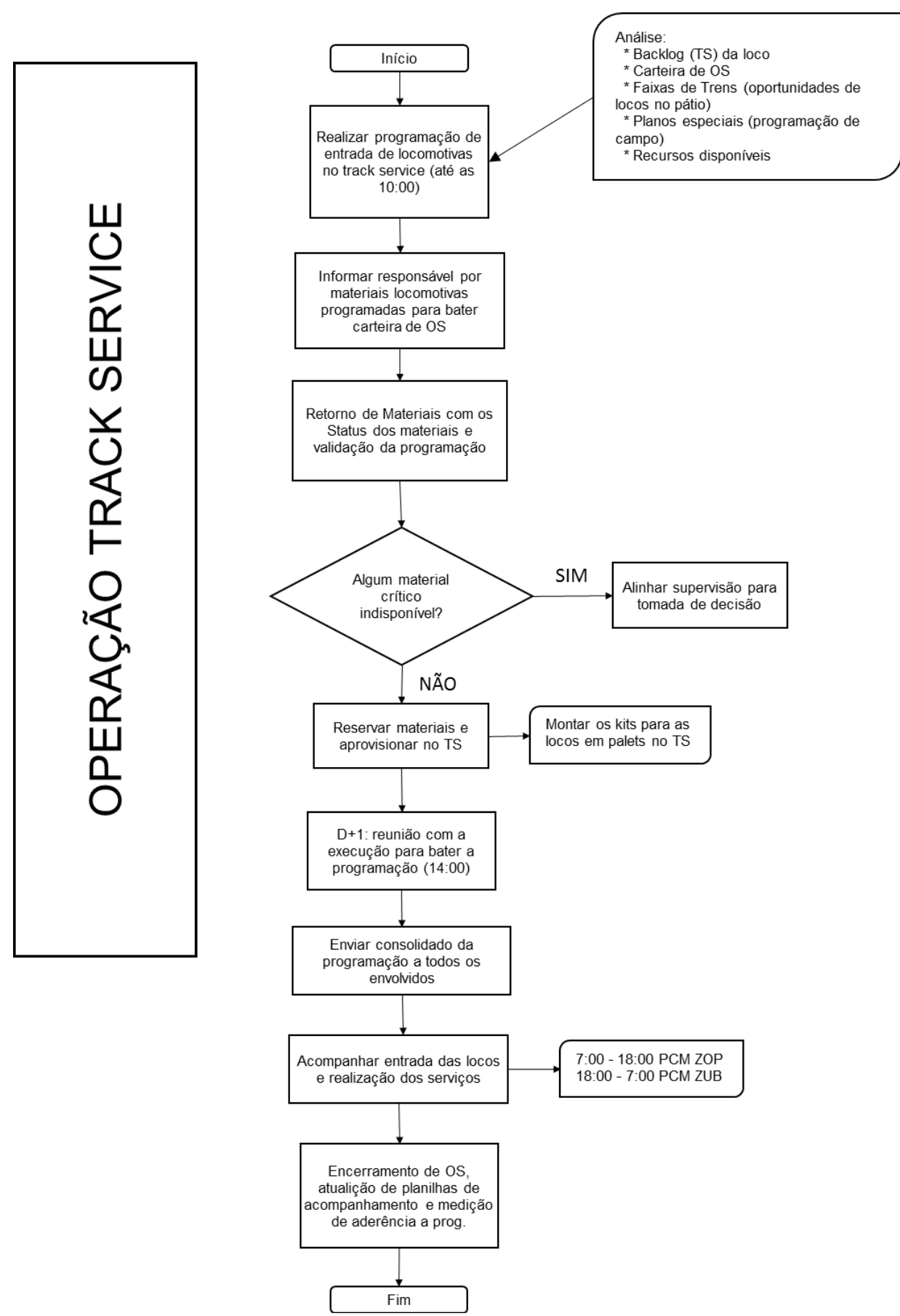

Figura 12. Fluxo do Padrão de PCM específico para o Track Service

Paralelamente à criação do padrão, foi elaborada a lista com 48 materiais básicos que deverão estar disponíveis no container de materiais. Foi definido também um nível mínimo para cada material, de forma que seja reposto os materiais consumidos diariamente pelo setor de armazém.

Para acompanhamento da evolução do novo processo "Track Service" é fundamental que existam indicadores de rotinas que sejam acompanhados periodicamente, visando corrigir desvios e mensurar os ganhos defendidos no início do projeto. Dessa forma, o PCM Centro Sudeste, consolida os indicadores de aderência à programação e backlog de ITS em um relatório semanal com os demais indicadores do corredor, conforme ilustrado nas figuras 13 e 14 . 
IV'S REALIZADAS NO TRACK SERVICE

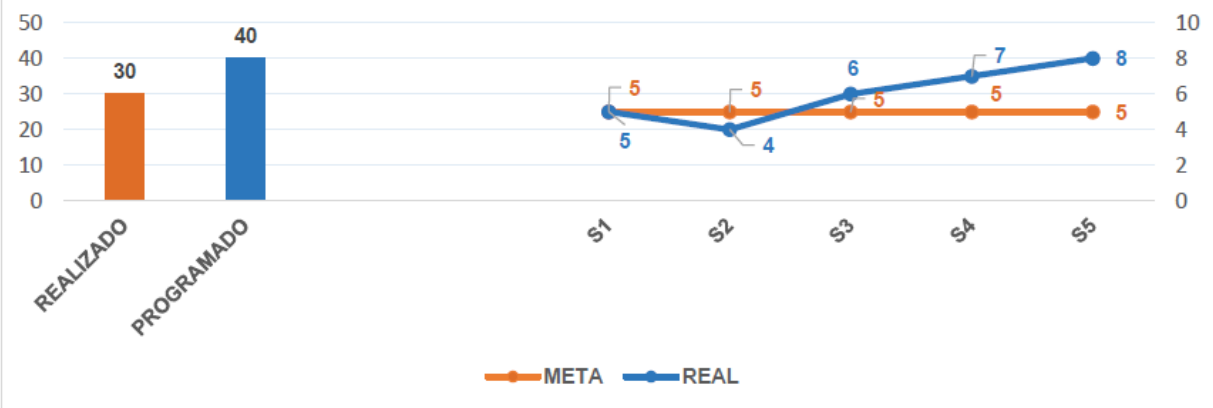

Figura 13. Aderência à programação de ITS

\begin{tabular}{|c|c|c|c|}
\hline LOCO & FROTA & Última IV & Backlog \\
\hline 8175 & SD70 & $15 / 10 / 2016$ & 50 \\
\hline 8194 & GT46 & $09 / 11 / 2016$ & 26 \\
\hline 8219 & GT46 & $21 / 10 / 2016$ & 45 \\
\hline 8220 & GT46 & $28 / 10 / 2016$ & 38 \\
\hline 8221 & GT46 & $02 / 11 / 2016$ & 33 \\
\hline 8246 & GT46 & $02 / 11 / 2016$ & 33 \\
\hline 8251 & GT46 & $09 / 11 / 2016$ & 26 \\
\hline 8254 & GT46 & $02 / 11 / 2016$ & 33 \\
\hline 8340 & SD70BB & $01 / 12 / 2016$ & 4 \\
\hline 8342 & SD70BB & $01 / 12 / 2016$ & 4 \\
\hline 8354 & SD70BB & $29 / 10 / 2016$ & 37 \\
\hline 8565 & AC44 & \begin{tabular}{|l}
$17 / 10 / 2016$ \\
\end{tabular} & 48 \\
\hline 8567 & AC44 & $16 / 11 / 2016$ & 18 \\
\hline 8569 & AC44 & $24 / 11 / 2016$ & 10 \\
\hline 8571 & AC44 & $17 / 11 / 2016$ & 17 \\
\hline 8573 & AC44 & $28 / 10 / 2016$ & 37 \\
\hline 8575 & AC44 & $16 / 11 / 2016$ & 18 \\
\hline 8576 & AC44 & $24 / 11 / 2016$ & 10 \\
\hline 8577 & AC44 & $02 / 12 / 2016$ & 3 \\
\hline 8578 & AC44 & $03 / 12 / 2016$ & 2 \\
\hline 8579 & AC44 & $17 / 11 / 2016$ & 17 \\
\hline 8580 & AC44 & $15 / 10 / 2016$ & 50 \\
\hline 8581 & AC44 & $23 / 11 / 2016$ & 11 \\
\hline 8582 & AC44 & $01 / 11 / 2016$ & 34 \\
\hline 8585 & AC44 & $23 / 11 / 2016$ & 11 \\
\hline 8588 & AC44 & $28 / 10 / 2016$ & 37 \\
\hline 8590 & AC44 & $02 / 12 / 2016$ & 3 \\
\hline 8592 & AC44 & $22 / 11 / 2016$ & 12 \\
\hline 8593 & AC44 & $20 / 11 / 2016$ & 14 \\
\hline 8594 & AC44 & $20 / 11 / 2016$ & 14 \\
\hline 8662 & SD70 & $27 / 11 / 2016$ & 7 \\
\hline 8663 & SD70 & $25 / 11 / 2016$ & 9 \\
\hline 8664 & SD70 & $22 / 11 / 2016$ & 12 \\
\hline
\end{tabular}

Figura 14. Backlog de ITS da frota no final do mês de novembro

\section{CONCLUSÃO}

A vala e estruturas auxiliares do "Track Service" de Paulínia se encontram operacionais desde o final do ano de 2016. Os processos específicos e de interface estão definidos e detalhados com os responsáveis, sendo possível até mesmo acompanhar a evolução através dos indicadores criados. Todos os ganhos operacionais (OPEX) e ganhos em investimento (CAPEX) já foram contabilizados para o ano de 2017, mostrando a viabilidade de um projeto simples com custo e tempo de retorno reduzido. 